\title{
Ein Jahr COVID-19-Pandemie in den Geriatrischen Gesundheitszentren der Stadt Graz. Herausforderungen und Chancen
}

Hartmann Jörg Hohensinner und Christina Eva Peyker

\section{Einleitung}

\subsection{Ausgangssituation}

Seit dem ersten bestätigten Fall Anfang Dezember 2019 in der chinesischen Provinz Hubei breitete sich das neuartige Coronavirus in nur wenigen Monaten weltweit aus. Österreich schien anfänglich nicht betroffen zu sein, am 25. Februar 2020 wurde aber auch hier die erste Infektion diagnostiziert. Mitte März 2020 ergriff die Bundesregierung aufgrund der "ersten Welle“ einschneidende Maßnahmen zur Eindämmung der Pandemie (Pollak et al. 2021). Seitdem ist etwa ein Jahr vergangen, in dem sich für die Menschen, die Gesellschaft, das Gesundheitssystem, die Wirtschaft und viele andere Lebensbereiche sehr viel verändert hat (Kessler/Guggenbühl 2021). Vor allem das Gesundheitssystem wird auf eine noch nie da gewesene Belastungsprobe gestellt (Hofmacher/Singhuber 2020). Das gesamte Personal steht vor vollkommen neuen organisatorischen, medizinischen, pflegerischen und ethischen Herausforderungen (Fromm 2020). Akutkrankenhäuser können aus Schutz vor Überlastung und der Konzentration auf Intensivbetten nicht akute Operationen verschieben oder diese in Privatkrankenhäuser auslagern. In einer Sonderkrankenanstalt wie der Albert-Schweitzer-Klink (ASK), als Teil der Geriatrischen Gesundheitszentren der Stadt Graz (GGZ), ist dies aufgrund von Hochrisikopatient"innen nicht möglich. Die GGZ bestehend aus einem Sonderkrankenhaus mit 325 stationären Betten, einer Tagesklinik und betreutem Wohnen sowie vier ausgelagerten Pflegeheimen, bietet eine Versorgung und Betreuung für geriatrische Patient*innen und Bewohner*innen, Patient*innen mit Demenz, palliativen Patient*innen und Patient"innen im Wachkoma an. Aufgrund der Vulnerabilität der zu betreuenden Patient*innen und Bewohner*innen benötigen diese jeden Tag die volle medizinische und pflegerische Aufmerksamkeit. Die größte Herausforderung für die GGZ bestand darin, mit dieser vollkommen neuen, noch nie dagewesenen Situati- 
on umzugehen. Zentrales Anliegen war es, trotz Pandemie Patient"innen und Bewohner*innen bestmöglich zu versorgen. Das bedeutete gleichzeitig vor einer COVID-19-Infektion zu schützen, aber auch die Betreuung nicht zu vernachlässigen. Weiters ging es darum die Mitarbeiter*innen vor einer Ansteckung zu schützen. Zusätzlich stellte sich die Frage, wie Angehörige trotz Besuchsverbot Beistand leisten können und Langeweile und Einsamkeit der Patient*innen und Bewohner*innen verhindert werden können. (Fromm 2020, 106; Schippinger et al. 2020, 411). Diese großen Herausforderungen zeigten auf, dass es in einer Krise wie der COVID-19Pandemie ein funktionierendes, ineinandergreifendes System benötigt, das eine schnelle Koordination und Kommunikation an den entsprechenden Schnittstellen schafft und dadurch ein rasches und vor allem angemessenes Handeln ermöglicht (Leinich 2021, 3).

\section{Die Mitarbeiter"innen in der Krise}

Für die Mitarbeiter*innen der GGZ, insbesondere für die Pflegekräfte, stellte die Pandemie eine noch nie dagewesene Situation dar. Die Ungewissheit wie es weitergeht, die Angst sich selbst mit dem Virus zu infizieren und die Gefahr, die das Virus für die Patient*innen und Bewohner*innen darstellt, war groß. Fast täglich gab es neue Informationen und Vorgaben, welche aufgrund der Schnelle oftmals nicht ins gesamte Team kommuniziert werden konnten. Trotz dieser Umstände leisteten die Mitarbeiter*innen in jeder Phase einen herausragenden Einsatz und waren jederzeit bereit Lösungsmöglichkeiten für die Herausforderungen zu finden. Sie mussten eine hohe Flexibilität aufweisen um rasch auf die neuen Bedingungen reagieren zu können. Um die Mitarbeiter*innen und damit auch die Patient*innen und Bewohner*innen bestmöglich vor dem Virus zu schützen wurde ein umfassendes Präventionskonzept, bestehend aus erweiterten Hygienemaßnahmen sowie einem Mitarbeiter*innen-Monitoring eingesetzt. (Hammer-Maier 2021, 3; Schwarz 2021, 122).

\subsection{Hygienemaßnabmen}

Mit dem Coronavirus gingen verstärkte Hygienemaßnahmen einher. Zuerst bestand die große Herausforderung darin, ausreichend Schutzmaterial zu organisieren, da dieses wegen der großen Nachfrage weltweit knapp war. Dem Management ist es aber gelungen nach und nach ausreichend 
Material zu beschaffen (Hermann 2021, 42). Da die ASK in den letzten Jahren sehr durch MRE (multiresistente Erreger) und weitere Infektionen (z. B. Influenza, Noroviren) gefordert war, herrschten bereits vor der Pandemie hohe hygienische Sicherheitsstandards. Dieses Vorwissen stellte in der Krise einen großen Vorteil dar. Die Präventions- und Schutzmaßnahmen der Bundesregierung konnten gut umgesetzt werden und es war Verlass auf das Verständnis und Wissen der Mitarbeiter*innen bezüglich der gesetzten Maßnahmen (Schippinger et al. 2020, 412). Alle Mitarbeiter"innen der GGZ wurden von Hygienefachkräften bezüglich der Hygienemaßnahmen geschult. Um besser zu den Mitarbeiter"innen durchzudringen, wurden außerdem Schulungsvideos sowie E-Learing-Kurse gestaltet die jederzeit am Sharepoint abrufbar waren (Pirstingeret al. 2020, 38-39).

\subsection{Mitarbeiter"innen-Monitoring}

\subsubsection{Gesundheitsselbstüberprüfung}

Um erste Krankheitssymptome frühzeitig zu erkennen wurde bereits im Zuge der ersten „Welle“ eine Gesundheitsselbstüberprüfung der Mitarbeiter*innen eingeführt. Im Zuge dieses Selbstassessments wurden die Mitarbeiter*innen dazu angehalten täglich zu Hause vor Dienstantritt den eigenen Gesundheitszustand zu überprüfen. Bei jeglicher Form respiratorischer Infekte oder Krankheitssymptomen wurden die Mitarbeiter*innen dazu angehalten nicht in den Dienst zu kommen und umgehend Vorgesetzte zu kontaktieren. Durch die umgehende Meldung dieser Mitarbeiter*innen konnten gezielte Testungen vorgenommen werden und das Risiko für eine Infektion in enger Zusammenarbeit mit dem Gesundheitsamt Graz, unter der Leitung von Dr. Eva Winter, schnell evaluiert werden (Pux et al. 2020; Hermann 2021).

\subsubsection{Mitarbeiter"innen-Screening}

Anfänglich wurde zur Testung der Mitarbeiter*innen eine Screening-Straße (Antigen-Test) sowie eine Testambulanz (Antigen-Test und PCR-Test) eingerichtet. Seit Februar 2021 wurde aufgrund des Hochrisikobereiches festgelegt, dass Mitarbeiter*innen ohne COVID-19-Symptome vor Dienstantritt ein Eigenscreening durchführen mussten. Das Testergebnis dieses Screenings durfte vor Dienstantritt nicht älter als 48 Stunden sein. Mit- 
arbeiter*innen mit verdächtigen Symptomen mussten sich hingegen in der Test-Ambulanz einem Test unterziehen. Alle Mitarbeiter*innen wurden darauf sensibilisiert die Screenings eigenverantwortlich durchzuführen. Die Bestätigung des Selbsttests erfolgte anhand des Vier-Augen-Prinzips. Am Sharepoint konnte die/der getestete Mitarbeiter*in ein Formular ausfüllen und an die kontrollierende Person senden. Diese konnte das Screening-Ergebnis dann innerhalb von sechs Stunden bestätigen. Die Bestätigung des Selbsttests wurde dann an die getestete Person gesendet. Ein negatives Testergebnis hatte keine weiteren Auswirkungen. Bei positiver Testung wurde die dienstverantwortliche Person kontaktiert und ein PCR-Test in der Testambulanz veranlasst (Knausz et al. 2021, 2-3). Die Selbsttestung wurde von den Mitarbeiter*innen sehr gut angenommen. Sie zeigte die große Selbstverantwortung und Selbstorganisation der Mitarbeiter*innen auf, welche durch eine agile Denkweise der GGZ ermöglicht wird (Hohensinner/Peyker 2020, 81).

Abbildung 1: Selbsttest Mitarbeiter*innen (Geriatrische Gesundheitszentren der Stadt Graz 2021)

\begin{tabular}{|c|c|c|c|}
\hline $\begin{array}{l}\text { Berei } \\
\text { GGZ }\end{array}$ & & SARS-CoV-2- Testung & $G R A$ \\
\hline Verar & ich: & & $\begin{array}{l}\text { GERIATRISCHE } \\
\text { GESUNDHEITSZENTREN }\end{array}$ \\
\hline stelle & & & \\
\hline ers.Nr. & Name: & & Datum: \\
\hline 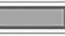 & $\square$ & & 07.05 .2021 \\
\hline & Bereich: & & \\
\hline & Pflegedi & & \\
\hline
\end{tabular}

\begin{tabular}{|l|}
\hline Test \\
\hline Testgruppe: \\
OMitarbeiterInnen \\
Testart: \\
Selbsttest \\
Mehrfachtest oder Drittperson \\
\hline
\end{tabular}

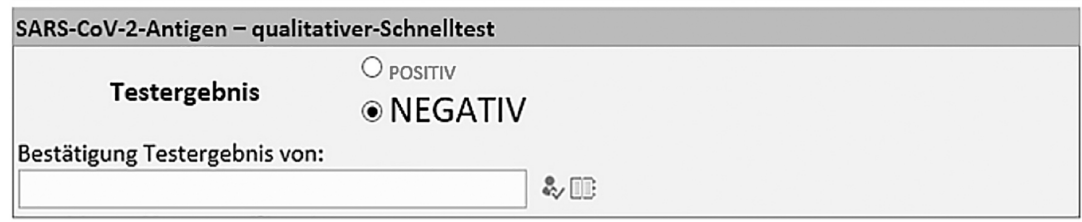




\subsection{Gegenseitige Unterstützung}

Durch Dienst-Freistellung von Mitarbeiter*innen aus Bereichen der Verwaltung oder der Therapie konnten wichtige Personalreserven aufgebaut werden, welche auch genutzt wurden. So unterstützten zum Beispiel Mitarbeiter"innen aus dem Human Resources Management, dem Qualitätsmanagement oder aus der Geschäftsführung die Pflegeheime der GGZ. Diese Mitarbeiter*innen standen vor der Herausforderung vollkommen neue Aufgaben übernehmen zu müssen. Dennoch war das gegenseitige Vertrauen und der Zusammenhalt enorm (Hermann 2021, 42; Schwarz 2021, 123). Diese Art der Job-Rotation brachte für die Mitarbeiter*innen aber auch Chancen in der Krise. Durch die Erfahrungen in anderen Bereichen der GGZ entstand ein umfassender Blick auf das Unternehmen. Es bestand die Möglichkeit sich auszutauschen und weiterzuentwickeln. Durch den Perspektivenwechsel konnten neue Ideen eingebracht werden um bestehende Abläufe und Prozesse zu optimieren (Tscherne 2014, 10).

\section{Der Schutz der uns vertrauenden Menschen}

Ältere, multimorbide und immungeschwächte Personen, wie unsere Patient*innen und Bewohner*innen, stellen eine sehr vulnerable Gruppe dar. Verschiedenste altersbedingte und immunologische Besonderheiten sowie eine bestehende Multimorbidität haben zur Folge, dass ältere Menschen ein hohes Risiko für schwere und letale Verläufe einer COVID-19-Erkrankung haben. Sie stellen daher eine besonders schutzbedürftige Risikogruppe dar (Sailer-Kronlechner 2021, 39). Für die GGZ war es das größte Anliegen, die ihnen vertrauenden Patient*innen und Bewohner*innen auch in dieser schwierigen Zeit bestmöglich zu betreuen. Die größte Herausforderung in diesem Zusammenhang bestand darin, den Patient"innen, Bewohner*innen, aber auch Angehörigen, die Notwendigkeit der Schutzmaßnahmen sowie der Besuchsbeschränkungen verständlich zu machen. Die Mitarbeiter*innen der GGZ waren in dieser Zeit stets darum bemüht, die ihnen vertrauenden Menschen bestmöglich zu schützen und zu begleiten, alternative Kontaktformen zu Angehörigen zu finden und trotz Pandemie Aktivitäten und Angebote gegen Langeweile und Einsamkeit zu ermöglichen (Geriatrische Gesundheitszentren der Stadt Graz 2020). 


\subsection{Screening der Patient"innen und Bewohner*innen}

Da die gesamte GGZ einen Hochrisikobereich darstellen, wurde für Patient"innen und Bewohner*innen eine engmaschige Teststrategie entwickelt. Die Patient*innen der ASK werden, unabhängig vom Impfstatus oder einer bereits erfolgten COVID-19-Erkrankung, in engmaschigen Zeitintervallen getestet. Bei stationärer Erst- oder Wiederaufnahme erfolgt ein Antigen- und PCR-Test in der Erstaufnahme (ERST). Am fünften und zehnten Tag nach Erst- oder Wiederaufnahme wird ein weiterer Antigentest und PCR-Tests durchgeführt. Weiters wird nach Kontakt mit COVID-19-positiven Personen am Tag eins, fünf, zehn und 14 ebenfalls ein Antigentest und PCR-Test durchgeführt. Zusätzlich wird den Patient*innen auf freiwilliger Basis einmal pro Woche ein Antigentest angeboten. Mit Ende März 2021 wurde diese Testfrequenz auf drei Mal pro Woche erhöht (Schmid et al. 2021, 2). Den Bewohner*innen der vier Pflegeheime der GGZ wird ebenfalls wöchentlich ein Antigen-Test angeboten. Bei Neuoder Wiederaufnahme von Bewohner*innen wird an Tag eins sowie nach drei bis fünf Tagen ein weiterer Antigen-Schnelltest durchgeführt. (Pojer 2021, 2).

Abbildung 2: COVID-19-Screening Patient*innen der ASK (Schmid et al. 2021, 2).

\begin{tabular}{|l|l|l|l|l|l|}
\hline & $\begin{array}{l}\text { Screening bei } \\
\text { stat. Erst- oder } \\
\text { Wiederauf- } \\
\text { nahme }\end{array}$ & $\begin{array}{l}\text { Screening am Tag } \\
\text { 5 und 10 nach } \\
\text { stat. Erst- oder } \\
\text { Wiederaufnahme } \\
\text { / Kontrolluntersu- } \\
\text { chung }\end{array}$ & $\begin{array}{l}\text { Screening } \\
\text { bei Besuch } \\
\text { der Tages- } \\
\text { Klinik/Ta- } \\
\text { geshospiz }\end{array}$ & $\begin{array}{l}\text { Screening bei po- } \\
\text { tentiell infektiösem } \\
\text { Kontakt mit COVID- } \\
\text { Pos. } \\
\text { Tag 1 } \\
\text { Tag 5 } \\
\text { Tag 10 } \\
\text { Tag 14 }\end{array}$ & $\begin{array}{l}\text { COVID-19 } \\
\text { Symptoma- } \\
\text { tik }\end{array}$ \\
\hline Pat. nicht geimpft & $\begin{array}{l}\text { Antigen und } \\
\text { PCR }\end{array}$ & Antigen und PCR & Antigen & Antigen und PCR & $\begin{array}{l}\text { Antigen und } \\
\text { PCR }\end{array}$ \\
\hline Pat. geimpft & $\begin{array}{l}\text { Antigen und } \\
\text { PCR }\end{array}$ & Antigen und PCR & Antigen & Antigen und PCR & $\begin{array}{l}\text { Antigen und } \\
\text { PCR }\end{array}$ \\
\hline St. p. COVID-19 & $\begin{array}{l}\text { Antigen und } \\
\text { PCR }\end{array}$ & Antigen und PCR & Antigen & Antigen und PCR & $\begin{array}{l}\text { Antigen und } \\
\text { PCR }\end{array}$ \\
\hline
\end{tabular}

\subsection{Umgang mit Besuchsbeschränkungen und reduzierten Kontaktmöglichkeiten}

Um Patient*innen und Bewohner*innen bestmöglich vor einer COVID-19-Infektion zu schützen wurden zunächst Besuche in allen Bereichen der GGZ verboten. Ausnahmen wurden nur in palliativen Situationen 
getroffen. Das Besuchsverbot galt für alle Personen, die nicht unmittelbar an der Aufrechterhaltung des Betriebes der ASK und den Pflegeheimen beteiligt waren. Das Betretungsverbot des Areals wurde von der Grazer Ordnungswache kontrolliert. Im April 2020 wurde dann ein Besuchscontainer bereitgestellt um kontrollierte und geschützte Kontaktmöglichkeiten für Patient"innen, Bewohner*innen und deren Besuch zu ermöglichen (Hermann 2021, 43). Die Besuchsbeschränkungen werden seitdem 14-tägig anhand eines Corona-Ampelsystems angepasst und evaluiert (Pirstinger et al. 2020, 2). Die GGZ konnten relativ schnell eine digitale Kontaktmöglichkeit zwischen Patient*innen, Bewohner*innen und deren Angehörigen ermöglichen. Es wurden rasch technische Voraussetzungen wie ein leistungsstarkes Internet sowie Tablets oder Handys organisiert. Am Franz Gerstenbrand Wachkoma Department der GGZ wurde vermehrt mit dem Augensteuerungs- und Kommunikationsgerät MyTobii gearbeitet um eine Kommunikation mit den Augen zu ermöglichen. Obwohl die Unterstützung der Patient"innen und Bewohner"innen bei der kontaktlosen Kommunikation eine große personelle Herausforderung darstellte, werden Medien wie MyTobii, Skype oder Telefonie zukünftig über die Pandemie hinaus wichtige alternative Kontaktmöglichkeiten darstellen (Kricheldorff 2020; Schaller 2021, 82).

\subsection{Alternative Aktivitäten und Angebote in der Krise}

Coronabedingte soziale Isolation, Langeweile und Einsamkeit können für ältere Menschen schwere negative Folgen für das Wohlbefinden und die Lebensqualität haben und gelten als Risikofaktoren für eine erhöhte Mortalität. Besuchsverbote und Kontaktbeschränkungen führen vor allem bei kognitiv beeinträchtigten älteren Menschen zu einer Veränderung gewohnter Rituale. Das gemeinsame Essen, Gruppenaktivitäten oder der Besuch der Familie sind nicht mehr möglich. Es entsteht ein Gefühl der Vereinsamung. In Folge dessen kann es zu Rückzug und Deprivation kommen. Für die GGZ stellte es eine große Herausforderung dar, einerseits die Patient*innen und Bewohner*innen vor einer COVID-19-Infektion zu schützen und andererseits auf die individuellen Bedürfnisse nach sozialen Kontakten, Zuwendung und Beschäftigung einzugehen. Ein großes Anliegen war es daher geeignete Lösungen und Kompromisse zu finden um eine Vereinbarkeit zwischen den individuellen Bedürfnissen der Patient"innen und Bewohner*innen und dem Interesse des Hauses zu ermöglichen (Halek et al. 2020, 52). Anhand der Corona-Ampel wurden die Aktivitäten je nach Lage ständig angepasst und evaluiert. Gewisse 
Angebote wurden in allen Phasen angeboten. Dazu zählten der Zugang zu Trainingsvideos, Teleberatung, therapeutische Distanzbetreuung, die kontaktlose Übernahme persönlicher Einkäufe, das Angebot von Hotlines (u. a. Psychologie, Seelsorge, Hospizverein), die Übertragung von Gottesdiensten über TV und Radio sowie das Streamen von Veranstaltungen auf TV-Geräte. Die berufsgruppenübergreifenden Angebote wurden ständig optimiert und werden teilweise auch nach der Pandemie fortgeführt werden (Pirstinger et al. 2020, 12).

\section{Die GGZ als agile Organisation in der Covid-19-Pandemie}

Die Corona-Pandemie bringt eine unsichere, sich ständig verändernde und dynamische Zeit für die GGZ mit sich. Umso mehr ist es in dieser Zeit wichtig flexibel, anpassungsfähig und schnell zu agieren um sich so auf unvorhersehbare Ereignisse und neue Anforderungen einstellen zu können (Böhm 2019, 9). Den GGZ ist dies durch eine agile Unternehmensgestaltung gelungen. In Zeiten der Pandemie war die Konzentration auf unvorhersehbare Ereignisse, die Fähigkeit der permanenten Anpassung, ein offensiver Umgang mit dem Wandel und eine schnelle Umsetzung von Maßnahmen sehr hilfreich. Weiters hat auch die gelebte Kreiskultur im Unternehmen ermöglicht mit der Pandemie besser umzugehen. In einer Kreiskultur wird gemeinsam Verantwortung getragen. Sie beruht auf Gleichwertigkeit, gegenseitigem Verständnis und einer gemeinsamen Suche nach Lösungen. Eine effektive Kommunikation sowie Wertehaltung und Loyalität stehen im Mittelpunkt (Rüther 2010, 112; Dittmar 2011). Agilität und Kreiskultur stehen für eine „Kultur der Gemeinsamkeit“ in diesen besonderen Zeiten (Häusling 2014). 
Abbildung 3: COVID-19 in den GGZ: Prozesse und Maßnabmen (eigene Darstellung angelehnt an Uhlmann et al. 2021, 9)

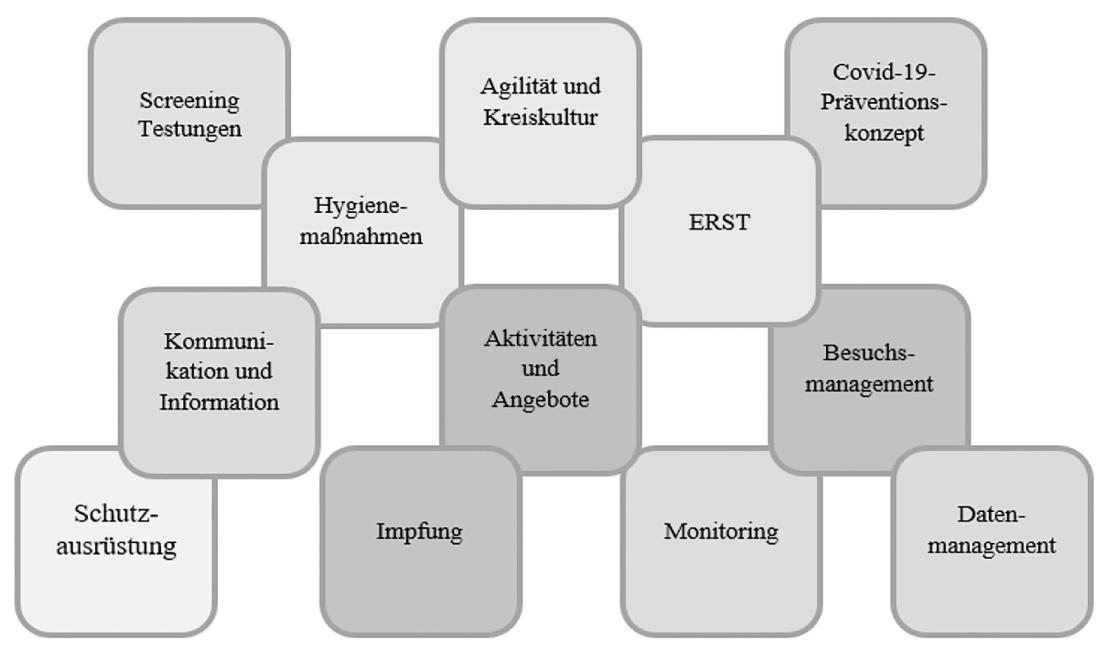

\section{Ein gemeinsamer Weg für ein Ziel}

Trotz der vollkommen neuen Situation war es der Organisation möglich Prozesse, die normalerweise durch die Qualitätssicherung und das Risikomanagement über einen langen Zeitraum hinweg entwickelt werden, an die neuen Bedürfnisse rasch anzupassen (Schippinger et al. 2020, 421).

\subsection{Informationsaustausch und Kommunikationswege}

Um allen Mitarbeiter*innen der GGZ relevante Informationen über das Corona-Virus zugänglich zu machen wurde im Intranet der GGZ eine "Corona Infoseite“ durch das Hygieneteam erstellt. Die Seite beinhaltet wichtige Informationen zu Hygiene, Erkrankungsfällen, Verdachtsfällen sowie dem Management von Fortbildungen. Weiters sind verschiedenste COVID-19-relevante Formulare abrufbar sowie Schulungsvideos bezüglich des Umgangs mit Schutzausrüstungen oder Testungen. Um die Mitarbeiter"innen vor einer gegenseitigen Ansteckung zu schützen, wurden außerdem alle Besprechungen als Video- oder Telefonkonferenz abgehalten. Zusätzlich wurde eine Hotline zu dienstrechtlichen, hygienerelevanten und medizinischen Fragen eingerichtet. Eine Helpline des psychologischen 
Teams der GGZ bietet zusätzlich Unterstützung für alle Mitarbeiter*innen an (Hermann 2021, 41-43).

\subsection{Datenmanagement und Datenerfassung}

Um einen Überblick über die unternehmensinterne COVID-19-Situation zu behalten, war ein zentrales Datenmanagement erforderlich. Aus diesem Grund wurde eine spezielle Datenbank eingerichtet, in der alle COVID-19relevanten Testdaten der gesamten GGZ erfasst und ausgewertet wurden. Durch diese Datenbank war es möglich, Daten über die Anzahl und Lokalisation der COVID-19-Verdachtsfälle, die Anzahl und Lokalisation positiv getesteter Patient"innen, Bewohner*innen und Mitarbeiter*innen, die Anzahl der ins Krankenhaus transferierten Personen und die Anzahl der sich aufgrund behördlicher Beschlüsse in Quarantäne befindlichen Mitarbeiter*innen zu erlangen. Dadurch konnten fundierte Entscheidungen über notwendige Maßnahmen getroffen werden. Zusätzlich wurden Daten über GGZ-interne Personalverschiebungen, Schlüsselpositionen und Absenzen erhoben und für das Management aufbereitet.

Bereits nach wenigen Wochen wurde zudem daran gearbeitet die Datenerhebung und -aufbereitung in die vorhandenen Informationssysteme (Krankenhausinformationssystem (KIS), Personalinformationssystem (PIS), Managementinformationssystem (MIS)) einzubinden um ein geeignetes Dashboard zur Verfügung zu haben und für kommende Situationen dieser Art gerüstet zu sein (Hohensinner 2021).

\subsection{Corona-Stab und Risikoanalyse}

Mit Beginn der Corona-Pandemie im März 2020 wurde in den GGZ ein Corona-Stab eingerichtet. In anfänglich täglichen und später dann wöchentlichen Telefonkonferenzen wurden auftretende Probleme im Zusammenhang mit COVID-19 aufgegriffen und strukturiert bearbeitet. Durch einen internen Newsletter wurden alle Mitarbeiter*innen kontinuierlich über wichtige Entscheidungen des Stabes informiert (Hermann 2021, 42). Mittels regelmäßiger Risikobewertungen in der ASK und in den Pflegeheimen wurden das COVID-19-Präventionskonzept sowie die Schutz- und Hygienemaßnahmen evaluiert (Uhlmann et al. 2021, 99). 


\subsection{COVID-19-Impfung}

Für die Durchführung der COVID-19-Schutzimpfung bei Mitarbeiter*innen der GGZ wurde ein Impfzentrum eingerichtet. Um einen effizienten Betrieb des Impfzentrums zu gewährleisten (Bsp.: Verfügbarkeit der notwendigen Anzahl an Impfdosen) hatten die Mitarbeiter*innen die Verpflichtung sich über das Intranet zu einem der acht Impftermine anzumelden. Durch das Terminmanagement und der Konzeption des Ablaufes der Impfungen im Impfzentrum war die Einhaltung der COVID-19-Präventionsmaßnahmen zu jeder Zeit sichergestellt. Auch für die Patient*innen und Bewohner*innen der GGZ werden laufend Impfungen angeboten (Knausz 2020, 3).

Seit Mai 2021 haben Mitarbeiter*innen ohne Impfung die Möglichkeit herauszufinden, ob in der Vergangenheit eine COVID-19-Infektion gegeben war. Dafür werden Antikörper-Test zur Selbsttestung angeboten (Langmann 2021).

\section{Schlussfolgerung und Ausblick}

Ereignisse wie die COVID-19-Pandemie sind schwer vorhersehbar oder beeinflussbar. Die Pandemie hat aufgezeigt, dass Infektionskrankheiten nicht überwunden sind, sondern auch in Zukunft eine sich schnell wandelnde Herausforderung darstellen können. Das oberste Ziel der GGZ war es, in dieser unsicheren und dynamischen Zeit ein sicheres Umfeld für die Patient*innen, Bewohner*innen, Angehörigen und Mitarbeiter*innen zu schaffen. Durch eine agile Denkweise und mit einer gemeinsam getragenen Verantwortung und einem großen Zusammenhalt ist es dem Unternehmen gelungen die Krise bis zum derzeitigen Zeitpunkt bestmöglich zu meistern. Die gesetzten Maßnahmen wurden gemeinsam getragen und es wurden viele entscheidende Schritte vorwärts unternommen, die über die Pandemie hinaus relevant sein werden. Für die Zukunft ist die GGZ gefordert ihr agiles Arbeiten bewusst und systematisch weiterzuentwickeln um für zukünftige Herausforderungen stets gewappnet zu sein. 


\section{Literatur}

Böhm, Janko: Erfolgsfaktor Agilität. Warum Scrum und Kanban zu zufriedenen Mitarbeitern und erfolgreichen Kunden führen, Wiesbaden: Springer Fachmedien 2019.

Dittmar, Vivian: Kreiskultur und Kommunikation. Grundform nachhaltiger Sozialstrukturen und Organisationsformen (2011), https://viviandittmar.net/wp-cont ent/uploads/kreiskultur-kommunikation.pdf [abgerufen am 05.05.2021].

Geriatrische Gesundheitszentren der Stadt Graz: Wie schützen wir die Schutzbedürftigsten in der Corona-Krise? (2020), https:/ggz.graz.at/de/Aktuelles/Presse/ Wie-schuetzen-wir-die-Schutzbeduerftigsten-in-der-Corona-Krise [abgerufen am 03.05.2021].

Geriatrische Gesundheitszentren der Stadt Graz: Selbst- und Abteilungstests 2021, https://mitarbeiter.graz.at/sites/ggz/SitePages/Homepage.aspx [abgerufen am 01.05.2021].

Halek, Margareta/Reuther, Sven/Schmidt, Jörg: Herausforderungen für die pflegerische Versorgung in der stationären Altenhilfe, in: Fortschritte der Medizin, 162/9 (2020) 51-53.

Hammer-Maier, Stefan: Nicht allein gelassen. Lockdown und Demenz, in: Geriatrie heute. Magazin der Geriatrischen Gesundheitszentren der Stadt Graz (2021) $3-12$.

Häusling, André: Die Relevanz agiler Personal- und Führungsinstrumente. Agile Führung als entscheidende Erfolgskomponente (2014), https://www.sigs-datac om.de/uploads/tx_dmjournals/Haeusling_OS_Agility_14.pdf [abgerufen am 05.05.2021].

Hermann, Brigitte: GGZ während der COVID-19-Pandemie, in: Geriatrie heute. Magazin der Geriatrischen Gesundheitszentren der Stadt Graz (2021) 41-43.

Hofmacher, Maria/Singhuber, Christopher: Leistungskraft regionaler Gesundheitssysteme in Zeiten von COVID-19, in: Health System Intelligence (2020) 1-102.

Hohensinner, Hartmann-Jörg: COVID-19 Testdaten. Geriatrische Gesundheitszentren der Stadt Graz 2021 [eigene Mitschrift].

Hohensinner, Hartmann-Jörg/Peyker, Christina: Ein Blick in den Pflegealltag am Department Franz-Gerstenbrand der Albert Schweitzer Klinik Graz in: Schaupp, Walter/Kröll, Wolfgang (Hrsg.): Spannungsfeld Pflege - Herausforderungen in klinischen und außerklinischen Settings, Baden-Baden: Nomos Verlag, 2020, 71-89.

Kessler, Claudia/Guggenbühl, Lisa: Auswirkungen der Corona-Pandemie auf gesundheitsbezogene Belastungen und Ressourcen der Bevölkerung (2021), https:/ /gesundheitsfoerderung.ch/assets/public/documents/de/5-grundlagen/publikatio nen/diverse-themen/arbeitspapiere/Arbeitspapier_052_GFCH_2021-01_-_Auswi rkungen_der_Corona-Pandemie.pdf [abgerufen am 28.03.2021].

Knausz, Iris: Organisationsrichtlinie Ablauforganisation COVID 19-Impfzentrum ASK, Geriatrische Gesundheitszentren der Stadt Graz (2020) 1-7. 
Knausz, Iris/Schmid, Marianne/Wiesinger, Tatjana/Pux Christian: Organisationsrichtlinie Ablauforganisation Mitarbeiter-Screening SARS-CoV-2 in der ASK, Geriatrische Gesundheitszentren der Stadt Graz (2021) 1-5.

Kricheldorff, Cornelia: Gesundheitsversorgung und Pflege für ältere Menschen in der Zukunft. Erkenntnisse aus der Corona-Pandemie, in: Zeitschrift für Gerontologie und Geriatrie, 3 (2020) 742-748.

Langmann, Daniela: Sondernewsletter Mai 2021, Geriatrische Gesundheitszentren der Stadt Graz 2021.

Leinich, Bernd: Vorwort, in: Geriatrie heute. Magazin der Geriatrischen Gesundheitszentren der Stadt Graz (2021) 9.

Pirstinger, Kerstin/Pojer, Martina/Sallegger, Andrea: COVID-19 Handbuch und Präventionskonzept Pflegewohnheime, Geriatrische Gesundheitszentren der Stadt Graz 2020, in: https://mitarbeiter.graz.at/sites/ggz/Hygiene/Dokument e\%20\%20COVID19/Handbuch\%20und\%20Pr\%C3\%A4ventionskonzept\%20P WHe.pdf [abgerufen am 06.04.2021].

Pojer, Martina: Testungen PWH BewohnerInnen und MitarbeiterInnen, Geriatrische Gesundheitszentren der Stadt Graz (2021) 2-3.

Pollak, Markus/Kowarz, Nikolaus/Partheymüller, Julia: Chronologie zur CoronaKrise in Österreich - Teil 1: Vorgeschichte, der Weg in den Lockdown, die akute Phase und wirtschaftliche Folgen, https:/viecer.univie.ac.at/corona-blog/c orona-blog-beitraege/blog51/ [abgerufen am 30.03.2021].

Pux, Christian/Uhlmann Michael/Knausz Iris: Gesundheitsselbstüberprüfung Personal GGZ, Geriatrische Gesundheitszentren 2020, 1, https://mitarbeiter.graz.at/ sites/ggz/Hygiene/Dokumente\%20\%20COVID19/Gesundheitsselbst\%C3\%BCbe rpr\%C3\%BCfung.pdf [abgerufen am 08.04.2021].

Rüther, Christian: Soziokratie. Ein Organisationsmodell. Grundlagen, Methoden und Praxis, o. O. 2010, 10-268 [Masterthesis Internet Universität].

Sailer-Kronlechner, Mona: COVID-19 und die Besonderheiten geriatrischer Patienten, in: Geriatrie heute. Magazin der Geriatrischen Gesundheitszentren der Stadt Graz (2021) 39-40.

Schaller, Eva Maria: Herausforderung Pandemie und Angehörige, in: Geriatrie heute. Magazin der Geriatrischen Gesundheitszentren der Stadt Graz (2021) 8283.

Schippinger, Walter/Pichler, Gerald/ Hohensinner, Hartmann-Jörg/Thonhofer, Annika/Gubisch, Karin: Der Umgang mit COVID-19 in einer Sonderkrankenanstalt. Erfahrungen der Albert-Schweitzer-Klinik als Teil der Geriatrischen Gesundheitszentren Graz (GGZ) in: Kröll, Wolfgang/Platzer, Johann/Ruckenbauer, Hans-Walter/Schaupp, Walter (Hrsg.): Die Corona-Pandemie. Ethische, gesellschaftliche und theologische Reflexionen einer Krise, Baden-Baden: Nomos Verlag, 2020, 407-422.

Schmid, Marianne/Pux Christian/Wiesinger Tatjana: Organisationsrichtlinie SARSCoV2-Testung Pat. und MA Albert Schweitzer Klinik, 6. Aktualisierte Auflage, Geriatrische Gesundheitszentren der Stadt Graz 2021, 1-3. 
Schwarz, Irene: Helfen ist Herzenssache. Das „Corona“-Jahr, in: Geriatrie heute. Magazin der Geriatrischen Gesundheitszentren der Stadt Graz, (2021) 122-125.

Tscherne, Anita: Human Resources Management - Personalentwicklungskonzept „Lebensphasenorientierte Unternehmensführung“, Geriatrische Gesundheitszentren der Stadt Graz (2014) 1-15.

Uhlmann, Michael/Pirstinger, Kerstin/Höhn, Martina: COVID-19: Ein Ziel - ein gemeinsamer Weg, in: Geriatrie heute. Magazin der Geriatrischen Gesundheitszentren der Stadt Graz (2021) 99. 